\title{
Quantum optomechanics with a mixture of ultracold atoms
}

\author{
H. Jing ${ }^{1,2}$, X. Zhao ${ }^{1}$, and L. F. Buchmann ${ }^{2}$ \\ ${ }^{1}$ Department of Physics, Henan Normal University, Xinxiang 45300\%, P.R. China \\ ${ }^{2}$ B2 Institute, Department of Physics and College of Optical Sciences, \\ The University of Arizona, Tucson, Arizona 85721
}

(Dated: August 18, 2018)

\begin{abstract}
We study cavity optomechanics of a mixture of ultracold atoms with tunable nonlinear collisions. We show that atomic collisions provide linear couplings between fictitious condensate oscillators, leading to possibilities of achieving a globally coupled quantum optomechanical network with an integrated atom chip. Potential applications range from simulating collective nonequilibrium dynamics in fields well past physics to probing unique properties of quantum mixtures.
\end{abstract}

PACS numbers: 42.50.-p, 03.75.Pp, 03.70.+k

Recent years have witnessed rapid advances in the field of cavity optomechanics [1 $[3]$. These advances have lead to striking demonstrations of quantum effects in mechanical objects at the mesoscale (single or two independent oscillators) [4], and opened up exciting new possibilities in developing integrated phononic circuits or coherent acoustic analogs of quantum nonlinear optics. Very recently, Lin et al. experimentally realized a direct mechanical coupling between two nano-oscillators and firstly observed remarkable effects of coherent mechanical wave mixing [5]. By establishing nearest-neighbor couplings in an optomechanical array [6], one can even synchronize vibrations of all elements [7]. For current nano-fabrication techniques, however, it remains a challenge to make a globally coupled optomechanical network, which has applications in simulating numerous important situations well past physics [8].

In parallel to the approach to cavity optomechanics that relies on the advanced materials and processing techniques of the semiconductor industry and nanoscience, an alternative approach relies on the realization of optomechanics where momentum sideband or particle-hole excitations of an atomic Bose-Einstein condensate (BEC) $[9$ 11] or a degenerate Fermi gas [12] play the role of the mechanical oscillator. In view of rapid advances in making and manipulating an ultracold mixture of two or more superfluids [13 18], here we probe the realization of multimode quantum acoustics [19], with linear mechanical couplings between the "BEC mirrors" arising from two-body atomic collisions. In contrast to the recently proposed nano-fabricated arrays with only nearest-neighbor couplings 7], our cold-atom system presents an interesting example of how to generate a multi-component quantum network with global couplings between different "mirror" modes.

We consider a cigar-shaped $n$-component BEC trapped in an optical Fabry-Pérot cavity of length $L$, with the soft trapping direction parallel to the cavity axis $z$ (see Fig. 1 ). We further assume that the trapping strength perpendicular to the cavity axis is sufficient to allow us to treat the system as quasi-1D, and to only consider excitations along the cavity axis 18. Within the dipole and rotating-wave approximations, the atomic part of Hamil-

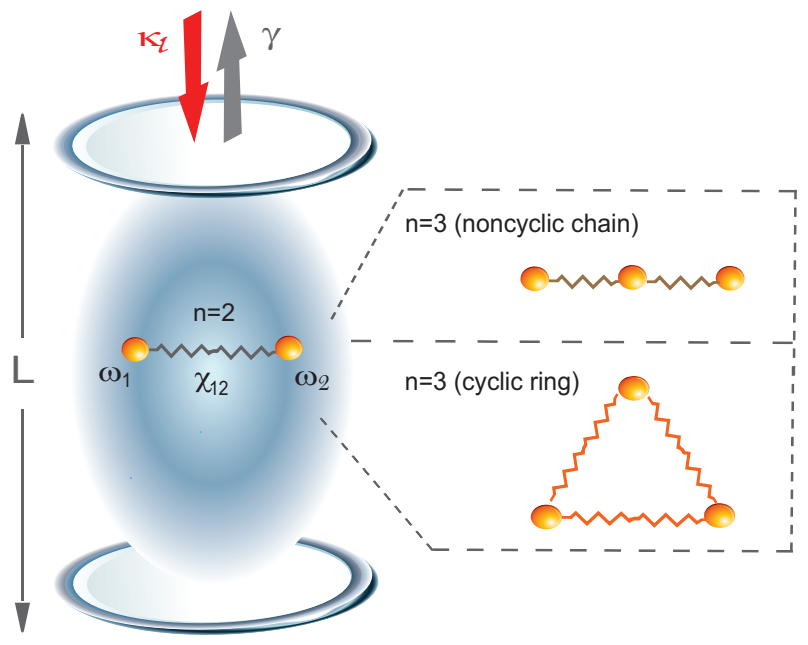

FIG. 1: (Color online) Quantum optomechanical network of an ultracold multi-species mixture. The tunable interspecies collisions provide a global coupling of fictitious mirrors, leading to collective mechanical excitations.

tonian is written

$$
\begin{aligned}
\hat{H} & =\int d z \sum_{i, j \neq i} \hat{\Psi}_{i}^{\dagger}(z)\left[\frac{\hat{P}_{i}^{2}}{2 M_{i}}+\hbar U_{i} \cos ^{2}(k z) \hat{c}^{\dagger} \hat{c}\right. \\
& \left.+\hbar \chi_{i}^{\prime} \hat{\Psi}_{i}^{\dagger}(z) \hat{\Psi}_{i}(z)+\hbar \frac{\chi_{i j}^{\prime}}{2} \hat{\Psi}_{j}^{\dagger}(z) \hat{\Psi}_{j}(z)\right] \hat{\Psi}_{i}(z) .
\end{aligned}
$$

Here the subscripts $i, j=1,2, \ldots, n$, denoting the species of bosonic atoms, $M_{i}$ is the atomic mass, $\hat{\Psi}_{i}$ is the atomic field operator, $\hat{c}$ is the annihilation operator of the cavity field, $k=\omega_{L} / c$ is the wave number of the pump laser, $U_{i}=g_{i}^{2} /\left(\omega_{L}-\omega_{i}^{\prime}\right)$ with $g_{i}$ the single-photon Rabi frequency and $\omega_{i}^{\prime}$ the atomic resonance frequency. The nonlinear collision term includes both intra-component and inter-component collisions $\left(\chi_{i j}^{\prime}=\chi_{j i}^{\prime}, j \neq i\right)$, which can be tuned separately via magnetic Feshbach resonance techniques 13 15].

Under the weak-excitation approximation the atomic field operator for each species can be expanded in terms of its zero-momentum component and its lowest order 
momentum side mode 9 ]

$$
\sqrt{L} \hat{\Psi}_{i}(z)=\sqrt{N_{i}}+\hat{b}_{i} \sqrt{2} \cos (2 k z),
$$

where $N_{i}$ the atom number for species $i$. The resulting Hamiltonian can be written in a form that describes a system of driven, coupled mechanical oscillators by mapping each atomic species to its own fictitious oscillator mode with displacement and momentum $\hat{Q}_{i}=$ $\left(\hat{b}_{i}^{\dagger}+\hat{b}_{i}\right) / \sqrt{2}$ and $\hat{P}_{i}=i\left(\hat{b}_{i}^{\dagger}-\hat{b}_{i}\right) / \sqrt{2}$, respectively. The condensate+cavity-field Hamiltonian is then

$$
\hat{H}=\hat{H}_{M}+\hat{H}_{O M} .
$$

where

$$
\begin{aligned}
\hat{H}_{M} & =\sum_{i, j \neq i} \hbar\left[\frac{\omega_{i}}{2}\left(\hat{P}_{i}^{2}+\hat{Q}_{i}^{2}\right)+\chi_{i} \hat{Q}_{i}^{2}+\frac{\chi_{i j}}{2} \hat{Q}_{i} \hat{Q}_{j}\right], \\
\hat{H}_{O M} & =-\hbar \delta \hat{c}^{\dagger} \hat{c}+i \hbar \eta\left(\hat{c}^{\dagger}-\hat{c}\right)+\sum_{i=1}^{n} \hbar g_{m, i} \hat{c}^{\dagger} \hat{c} \hat{Q}_{i} .
\end{aligned}
$$

Here

$$
g_{m, i}=\frac{g_{i}^{2}}{2\left(\omega_{L}-\omega_{i}^{\prime}\right)} \sqrt{N_{i}}
$$

is the optomechanical coupling between the cavity field and the condensate side modes, and

$$
\delta=\omega_{\mathrm{L}}-\omega_{\mathrm{C}}-\sum_{i=1}^{n} \sqrt{N_{i}} g_{m, i}
$$

is the detuning of the pump laser frequency $\omega_{\mathrm{L}}$ with respect to the sum of the empty cavity resonance frequency $\omega_{\mathrm{C}}$ shifted by the presence of the atoms. Also, $\omega_{i}=$ $2 \hbar k^{2} / M_{i}+2 N_{i} \chi_{i}^{\prime} / L+N_{j} \chi_{i j}^{\prime} / L, \chi_{i}=2 N_{i} \chi_{i}^{\prime} / L, \chi_{i j}=$ $2\left(N_{i} N_{j}\right)^{1 / 2} \chi_{i j}^{\prime} / L$, and $\eta$ is the pump rate of the cavity. A formally similar picture of linearly coupled oscillators can also be achieved for a Fermi-Fermi or Bose-Fermi mixture trapped in a cavity [12]. In addition, a full description of the system also includes loss mechanisms, most importantly here the cavity decay, which is described as usually by coupling to a reservoir, a term not shown explicitly here.

The role of atomic collisions is three-fold: firstly, a frequency shift from the recoil one: $2 \hbar k^{2} / M_{i} \rightarrow \omega_{i}$; secondly, the mechanical self-squeezing due to $\chi_{i}^{\prime}$; thirdly and most importantly, the atomic collisions create global linear couplings $\hat{H}_{\text {global }}=\hbar \sum_{i \neq j} \frac{\chi_{i j}}{2} \hat{Q}_{i} \hat{Q}_{j}$ between the BEC mirrors, providing considerable flexibility in controlling the dynamics of the system [20, 21]. In contrast to nano-fabricated optomechanical arrays with local couplings [7], ultracold atomic mixtures provide a system which can be explored for a globally coupled phononic network.

We proceed by writing the Heisenberg-Langevin equa- tions $(\hbar=1)$

$$
\begin{aligned}
\frac{d \hat{c}}{d t} & =-i \sum_{i=1}^{n}\left(\delta+g_{m, i} \hat{Q}_{i}\right) \hat{c}+\eta-\gamma \hat{c}+\sqrt{2 \kappa} \hat{c}_{\mathrm{in}}, \\
\frac{d \hat{Q}_{i}}{d t} & =\sum_{j \neq i} \omega_{i j} \hat{P}_{i}, \\
\frac{d \hat{P}_{i}}{d t} & =-\left(\omega_{i j}+2 \chi_{i}\right) \hat{Q}_{i}-\sum_{j \neq i} \frac{\chi_{i j}}{2} \hat{Q}_{j}-g_{m, i} \hat{c}^{\dagger} \hat{c},
\end{aligned}
$$

and similarly for $\left(\hat{Q}_{j}, \hat{P}_{j}\right)$, with $(i, j) \rightarrow(j, i)$. Here $\kappa$ is the cavity decay rate and $\hat{c}_{\text {in }}$ is the associated cavity gaussian noise operator, with zero mean and two-time correlations $\left\langle\hat{c}_{i n}(t) \hat{c}_{i n}^{\dagger}\left(t^{\prime}\right)\right\rangle=\delta\left(t-t^{\prime}\right)$ and $\left\langle\hat{c}_{i n}(t) \hat{c}_{i n}\left(t^{\prime}\right)\right\rangle=$ 0 . The condensate losses are neglected here since they are significantly slower than cavity losses.

The mean-field solutions of the Langevin equations can be obtained by setting all time derivatives be zero. For the dual-species BEC case $(n=2)$, we have

$$
\begin{aligned}
c_{s} & =\frac{\eta}{\gamma+i\left(\delta+g_{m, 1} Q_{1}^{s}+g_{m, 2} Q_{2}^{s}\right)}, \quad P_{i}^{s}=0, \\
Q_{i}^{s} & =\frac{\left[\left(\omega_{(3-i)}+2 \chi_{(3-i)}\right) g_{m, i}-\chi_{12} g_{m,(3-i)}\right]\left|c_{s}\right|^{2}}{\chi_{12}^{2}-\left(\omega_{1}+2 \chi_{1}\right)\left(\omega_{2}+2 \chi_{2}\right)} .
\end{aligned}
$$

With this approach, the optomechanical coupling between the "mirror" and the cavity can be linearized in the shifted basis, i.e. $\hat{\mathcal{H}}_{O M}=\sum_{i=1}^{n} g_{m, i}\left|c_{s}\right|^{2} \hat{Q}_{i}$. In comparison to previous works on independent mirrors [4], the inter-species collisions bring fundamental impacts on the properties of the system. For example, with $a_{12} \sim 0$ [16], we have: $Q_{i}^{s}=-g_{m, i}\left|c_{s}\right|^{2} /\left(\omega_{i}+2 \chi_{i}\right)[9]$. By tuning interspecies collisions, we can realize coherent mechanical swapping between $Q_{1}^{s}=0, Q_{2}^{s} \neq 0$ and $Q_{2}^{s}=0, Q_{1}^{s} \neq 0$, which otherwise is unaccessible.

The general $n$-component BEC case is much simplified by assuming $M_{i}=M, N_{i}=N, \chi_{i}=\chi, \chi_{i j}=\tilde{\chi}, g_{m, i}=$ $g_{m}$, and $\omega_{i j}=\omega$, in which case we simply have

$$
Q_{i}^{s}=-\frac{g_{m}\left|c_{s}\right|^{2}}{\omega+2 \chi+(n-1) \tilde{\chi}}, \quad(i=1,2, \ldots, n)
$$

indicating global amplitude synchronization of the BEC "network". In contrast, for an array of mirrors with only nearest-neighbor couplings, we have $(n=3): Q_{1,3}^{s}=$ $(\tilde{\chi}-\omega-2 \chi) g_{m}\left|c_{s}\right|^{2} /\left[(\omega+2 \chi)^{2}-2 \tilde{\chi}^{2}\right]$, and $Q_{2}^{s}-Q_{1,3}^{s}=$ $\tilde{\chi} g_{m}\left|c_{s}\right|^{2} /\left[(\omega+2 \chi)^{2}-2 \tilde{\chi}^{2}\right] \neq 0$, i.e. neither universal relation for $Q_{i}^{s}$ nor global amplitude synchronization can exist for this case. We have also studied other noncyclic or cyclic arrays $(n \geq 4)$ with only nearest-neighbor linear couplings, and found similar features like this.

Now we study the role of collisions on quantum normalmode behaviors of coupled BEC mirrors. For $\gamma \gg \omega_{i}$ the cavity field, approaching its steady state in a timescale $\gamma^{-1}$, follows adiabatically the mirrors positions. In this case the mechanical dynamics are robust against the cavity field fluctuations, and we can treat the optical field 
classically. The resulting effective Hamiltonian describes a model of coupled driven oscillators, i.e.

$$
\begin{aligned}
& \hat{\mathcal{H}}_{\text {eff }}=\sum_{i=1}^{n} \hat{\mathcal{H}}_{i}+\hat{H}_{\text {global }}, \\
& \hat{\mathcal{H}}_{i}=\frac{\omega_{i}}{2}\left(\hat{P}_{i}^{2}+\hat{Q}_{i}^{2}\right)+\chi_{i} \hat{Q}_{i}^{2}+g_{m, i} \bar{I} \hat{Q}_{i}
\end{aligned}
$$

with the intracavity intensity $\bar{I} \approx \frac{\eta^{2}}{\gamma^{2}+\delta^{2}}$ for $Q_{i}^{s} \ll \sqrt{N_{i}}$. For the dual-species BEC, this model is exactly solvable by defining new variables

$$
\left(\begin{array}{l}
\hat{q}_{1} \\
\hat{q}_{2}
\end{array}\right)=\left(\begin{array}{cc}
\omega_{1}^{-\frac{1}{2}} R_{+} & -\omega_{2}^{-\frac{1}{2}} R_{-} \\
\omega_{1}^{-\frac{1}{2}} R_{-} & \omega_{2}^{-\frac{1}{2}} R_{+}
\end{array}\right)\left(\begin{array}{l}
\hat{Q}_{1} \\
\hat{Q}_{2}
\end{array}\right)
$$

and

$$
\left(\begin{array}{l}
\hat{p}_{1} \\
\hat{p}_{2}
\end{array}\right)=\left(\begin{array}{cc}
\sqrt{\omega_{1}} R_{+} & -\sqrt{\omega_{2}} R_{-} \\
\sqrt{\omega_{1}} R_{-} & \sqrt{\omega_{2}} R_{+}
\end{array}\right)\left(\begin{array}{c}
\hat{P}_{1} \\
\hat{P}_{2}
\end{array}\right)
$$

with $R_{ \pm}^{2}=\frac{1}{2}\left[1 \pm\left(4 \chi_{12}^{2} \omega_{1} \omega_{2}+\omega_{-}^{2}\right)^{-1 / 2} \omega_{-}\right]$, and $\omega_{\mp}=$ $\omega_{1}\left(\omega_{1}+2 \chi_{1}\right) \mp \omega_{2}\left(\omega_{2}+2 \chi_{2}\right)$, resulting in a decoupled system

$$
\hat{\mathcal{H}}_{e f f}=\sum_{i=1,2}\left[\frac{1}{2}\left(\hat{p}_{i}^{2}+\Omega_{i}^{2} \hat{q}_{i}^{2}\right)+\nu_{i} \bar{I} \hat{q}_{i}\right]
$$

where the normal-mode frequencies as a function of $\chi_{12}$ are

$$
\Omega_{1,2}\left(\chi_{12}\right)=\sqrt{\frac{1}{2}\left(\omega_{+} \mp \sqrt{\omega_{-}^{2}+4 \chi_{12}^{2} \omega_{1} \omega_{2}}\right)},
$$

and

$$
\nu_{1,2}=g_{m, 1} \sqrt{\omega_{1}} R_{ \pm} \mp g_{m, 2} \sqrt{\omega_{2}} R_{\mp} .
$$

The energy spectrum of the system simply reads $E_{n_{1}, n_{2}}=$ $\sum_{i=1,2} \hbar\left[\left(n_{i}+1 / 2\right) \Omega_{i}-\bar{I}^{2} \nu_{i}^{2} / 2 \Omega_{i}\right]$. The single-phonon transition of mechanical states, e.g. $|1,0\rangle,|0,1\rangle \leftrightarrow|0,0\rangle$ with the energy splitting $\hbar \Omega_{1,2}$, can be detected by measuring the photons emitted from the cavity.

The effect of $\chi_{12}$ on the splitting of normal-mode frequencies is significant even for $n=2$, see Fig. 2, where we have chosen the quasi-1D collision parameters [12, 16]

$$
\chi_{i i}^{\prime}=\frac{2 \hbar a_{i}}{M_{i} a_{i, \perp}^{2}} \frac{1}{1-C a_{i} / a_{i, \perp}} \simeq 2 a_{i} \omega_{\perp}, \quad \chi_{12}^{\prime} \simeq a_{12} \omega_{\perp}
$$

where $C=1.0326, a_{i, \perp}=\sqrt{\hbar /\left(M_{i} \omega_{\perp}\right)}, a_{12, \perp}=$ $\sqrt{\hbar /\left(M_{12} \omega_{\perp}\right)}, M_{12}=M_{1} M_{2} /\left(M_{1}+M_{2}\right)$, and $a_{i, \perp}, a_{12, \perp}$ are transverse (perpendicular to the cavity axis) oscillator lengths corresponding to the transverse trapping frequency $\omega_{\perp}$. Upon inspection, one might be concerned with the divergence of the collision parameters that occurs when $a_{i, \perp}=C a_{i}$ [24]. However, this case can be safely ignored in the present analysis. This becomes clear when one considers the parameters of stable ${ }^{87} \mathrm{Rb}-{ }^{41} \mathrm{~K}$
BECs. Since $M_{1}=87 u_{0}, M_{2}=41 u_{0}, u_{0} \sim 1.7 \times 10^{-27} \mathrm{~kg}$, $a_{1} \sim 99 a_{0}, a_{2} \sim 60 a_{0}, a_{12} \sim 163 a_{0}, a_{0}=0.53 \AA$, and $\omega_{\perp} \sim 2 \pi \times 3 \mathrm{kHz}$, we have $a_{i, \perp} / a_{i} \gg 1, a_{12, \perp} / a_{12} \gg 1$, i.e. being far from divergence regimes. Also, for the achievable laser wavelength $\lambda=500 \mathrm{~nm}$, and $N_{1,2}=1.2 \times 10^{5}$, $L=178 \mu m$ [9], $\gamma \sim 2 \pi \times 1.3 \mathrm{MHz}$, the shifted mechanical frequency shifts are far smaller than cavity decaying: $\omega_{1} \sim 0.07 \gamma, \omega_{2} \sim 0.09 \gamma$, well satisfying adiabatic approaches of the cavity field.
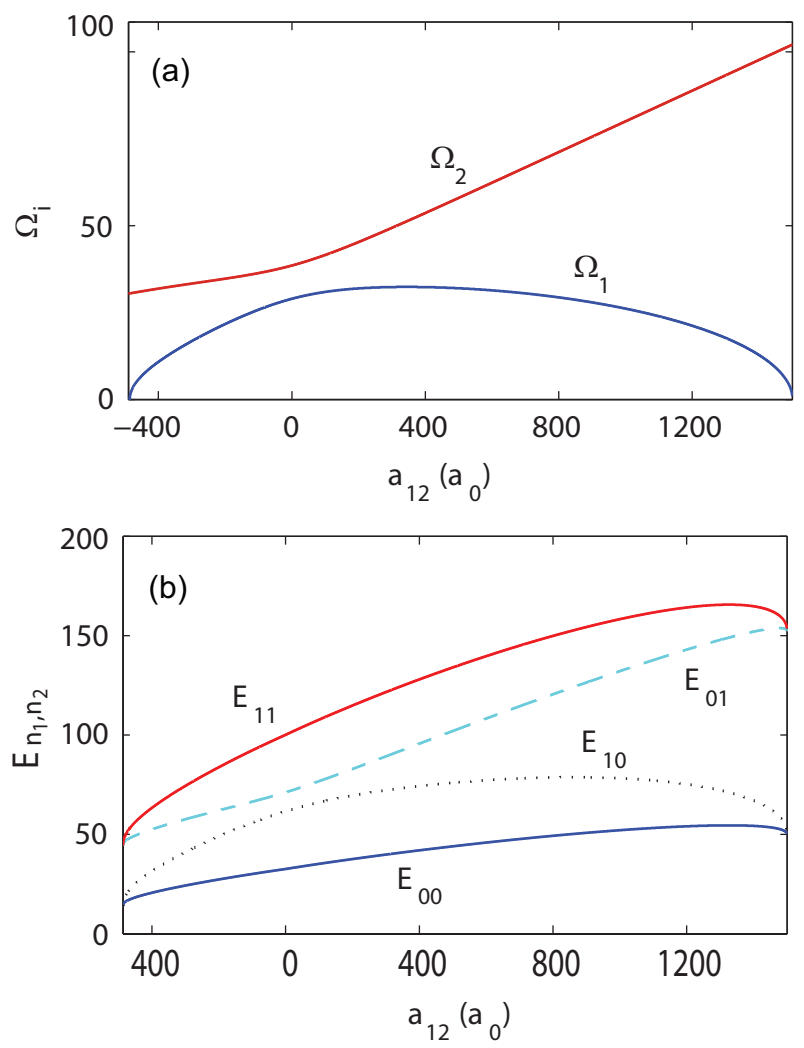

FIG. 2: (Color online) (a) The normal-mode splitting for coupled BEC mirrors (scaled by $\omega_{\perp}$ ); (b) the low-lying energy spectrum of the system, where we have chosen $D_{2}$-line transitions for both species atoms i.e. $g_{1,2} \sim 2 \pi \times 10.9 \mathrm{MHz}$, $\left(\omega_{L}-\omega_{1,2}^{\prime}\right) \sim 2 \pi \times 32 \mathrm{GHz}$, and $\left(\omega_{L}-\omega_{C}\right) \sim 2 \pi \times 200 \mathrm{MHz}$.

For a three-component mixture $(n=3)$ [12, 18], the resulting linear model of a tripartite quantum mechanical "ring" can be similarly solved. For simplicity we take $\chi_{i}=0, \chi_{i j}=\tilde{\chi}$, and $\omega_{i j}=\omega$, the normal-mode frequencies of this system are then: $\Omega_{1}=\sqrt{\omega(\omega+2 \tilde{\chi})}$, $\Omega_{2,3}=\sqrt{\omega(\omega-\tilde{\chi})}$, or a single collective mode for $\tilde{\chi}=\omega$. This is quite different from that of a noncyclic array, i.e. $\Omega_{1}=\sqrt{\omega(\omega+\sqrt{2} \tilde{\chi})}, \Omega_{2}=\omega$, and $\Omega_{3}=\sqrt{\omega(\omega-\sqrt{2} \tilde{\chi})}$, which never arrive at a single nonzero value for $\tilde{\chi} \neq 0$. For $n \geq 4$, more exotic phononic structures can be realized, e.g. a star-like cluster of three arrays, a cyclic ring coupled with a noncyclic array, or two coupled rings (see Fig. 1), with possible applications in e.g. simulating a crystalline cavity or quantum computing [23]. 


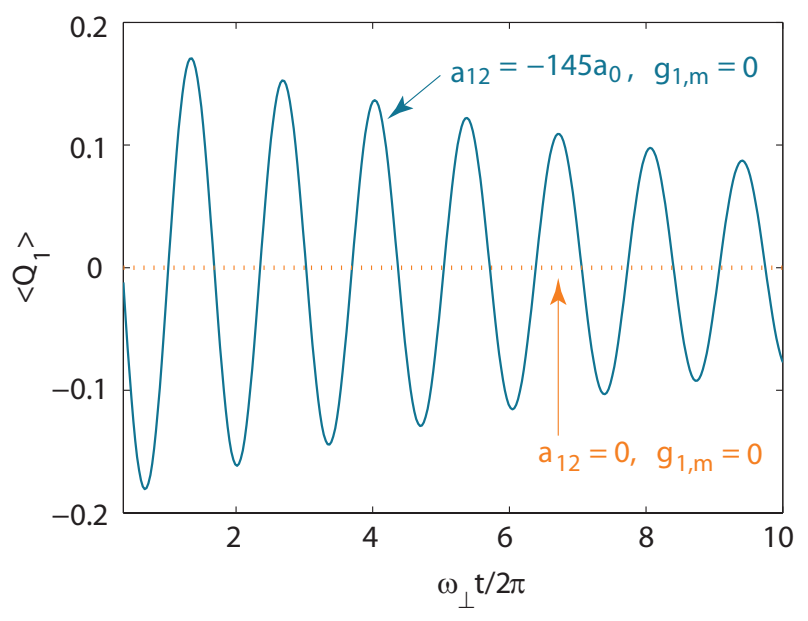

FIG. 3: (Color online) Even for $g_{1, m}=0$, coherent mechanical oscillations of $\left\langle Q_{1}\right\rangle$ can emerge due to nonzero values of $a_{12}$. The inter-species value $a_{12}$ can be tuned separately via magnetic Feshbach resonance techniques [13 15].

Finally we remark that besides the wide tunability of atomic collisions 13 15], one can also change the ratio of the interactions of light and different species atoms. As a specific example, for Rb-K mixtures, we can apply a species-selective dipole laser beam tuned to an inter- mediate wavelength between the $D_{1}$ and $D_{2}$ lines of $\mathrm{Rb}$ [22], leading to $g_{1, m} / g_{2, m} \ll 1$ since the dipole forces on $\mathrm{Rb}$ due to these two transitions cancel out. In this case $\chi_{12}$ provide an unique mechanical switch for the system from the single-mirror $\left(\chi_{12}=0\right)$ to the double-mirror $\left(\chi_{12} \neq 0\right)$ cases (see Fig. 3$)$.

In conclusion, we have investigated the realization of coupled quantum oscillators through a degenerate gas consisting of two different atomic species. We found that in contrast to intuitions, nonlinear atomic collisions provide a global linear coupling which facilitates mechanical mixing of two species. This is fundamentally different from that of independent cavity mirrors [4]. In view of rapid advances in experiments of ultracold mixtures [14 18], multi-species optomechanics are expected to be achievable in not too far future, with applications ranging from multimode quantum acoustics [19] to highprecision cavity diagnosis of ultracold collisions. Future studies will include hybrid-dimensional BEC mirrors 25], cooperative emissions of cavity photons from ultracold mixtures, and full quantum optomechanical control over Bose-Fermi mixtures.

We are grateful to P. Meystre for many helpful discussions and to supports from the NSFC (10974045 and 11274098), the Henan Chuangxin Plan, and the Y.T. Fok foundation.
[1] M. Aspelmeyer, S. Gröblacher, K. Hammerer, and N. Kiesel, J. Opt. Soc. Am. B 27, A189 (2010); T. J. Kippenberg and K. J. Vahala, Science 321, 1172 (2008); F. Marquardt and S. M. Girvin, Phys. 2, 40 (2009); I. Favero and K. Karrai, Nature Photon. 3, 201 (2009).

[2] J. Chan, et al. Nature (London) 478, 89 (2011); J. D. Teufel, et al. ibid. 475, 359 (2011); A. D. O'Connell, et al. ibid. 464, 697 (2010).

[3] E. Verhagen, et al. Nature (London) 482, 63 (2012); S. Kolkowitz, et al. Science 335, 1603 (2012); A. H. SafaviNaeini, et al. Phys. Rev. Lett. 108, 033602 (2012).

[4] M. Paternostro, G. D. Chiara, and G. M. Palma, Phys. Rev. Lett. 104, 243602 (2010); M. J. Hartmann and M. B. Plenio, ibid. 101, 200503 (2008).

[5] Q. Lin, et al. Nature Photon. 4, 236 (2010).

[6] M. Eichenfield, et al. Nature (London) 4, 236 (2010); D. E. Chang, A. H. Safavi-Naeini, M. Hafezi, and O. Painter, New J. Phys. 13, 023003 (2011).

[7] G. Heinrich, et al. Phys. Rev. Lett. 107, 043603 (2011); M. Ludwig and F. Marquardt, arXiv:1208.0327v1 (2012).

[8] M. G. Rosenblum and A. S. Pikovsky, Phys. Rev. Lett. 92, 114102 (2004); K. A. Takeuchi, et al. ibid. 107, 124101 (2011).

[9] F. Brennecke, S. Ritter, T. Donner, and T. Esslinger, Science 322, 235 (2008).

[10] D. M. Stamper-Kurn, arXiv:1204.4351v1 (2012); T. P. Purdy, et al. Phys. Rev. Lett. 105, 133602 (2010).

[11] N. Brahms, et al. Phys. Rev. Lett. 108, 133601 (2012).

[12] R. Kanamoto and P. Meystre, Phys. Rev. Lett. 104, 063601 (2010); Q. Sun, et al. Phys. Rev. A 84, 023822 (2011).
[13] G. Thalhammer, et al. Phys. Rev. Lett. 100, 210402 (2008).

[14] S. Ospelkaus, et al. Phys. Rev. Lett. 97, 120403 (2006).

[15] A. Simoni, et al. Phys. Rev. Lett. 90, 163202 (2003).

[16] S. B. Papp, J. M. Pino, and C. E. Wieman, Phys. Rev. Lett. 101, 040402 (2008).

[17] F. Crepin, G. Zarand, and P. Simon, Phys. Rev. Lett. 105, 115301 (2010); A. Trenkwalder, et al. ibid. 106, 115304 (2011).

[18] M. Taglieber, et al. Phys. Rev. Lett. 100, 010401 (2008); T. Fukuhara, S. Sugawa, and Y. Takahashi, Phys. Rev. A 76, 051604(R) (2007); M. Eto and M. Nitta, arXiv:1201.0343v1 (2012).

[19] H. Seok, L. F. Buchmann, S. Singh, and P. Meystre, arXiv:1208.5821 1 (2012); M. Abdi, A. R. Bahrampour, and D. Vitali, arXiv:1208.0915 2 (2012).

[20] K. B. Brown, et al. Nature (London) 471, 196 (2011); M. Harlander, et al. ibid. 471, 200 (2011).

[21] S. Gröblacher, K. Hammerer, M. R. Vanner, and M. Aspelmeyer, Nature (London) 460, 724 (2009); L. Tian and H. Wang, Phys. Rev. A 82, 053806 (2010); V. Fiore, et al. Phys. Rev. Lett. 107, 133601 (2011).

[22] C. Presilla and R. Onofrio, Phys. Rev. Lett. 90, 030404 (2003); J. Catani, et al. ibid. 103, 140401 (2009).

[23] E. K. Lindmark, et al. Opt. Lett. 21, 994 (1996); H. T. $\mathrm{Ng}$ and F. Nori, arXiv:1007.4338v1 (2010).

[24] M. Olshanii, Phys. Rev. Lett. 81, 938 (1998); E. Haller, et al. ibid. 104, 153203 (2010).

[25] G. Lamporesi, et al. Phys. Rev. Lett. 104, 153202 (2010). 